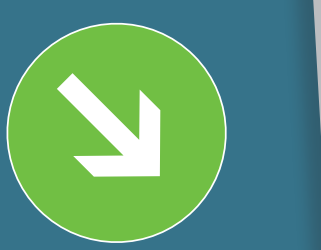

Ключевые слова: роботизированные системы, отделочная обработка, качество поверхности

\section{РОБОТИЗАЦИЯ ОТДЕЛОЧНОЙ ОБРАБОТКИ ДЕТАЛЕЙ В МАШИНОСТРОЕНИИ}

\author{
Михаил ВАРТАНОВ, Алексей ВЛАСОВ
}

Рассмотрены перспективы применения промышленных роботов для выполнения механической отделочной обработки деталей в машиностроении. Приведены схемы построения операций с использованием роботов. Приведен состав ячейки для выполнения роботизированной отделочной обработки. Изложены результаты экспериментальных исследований, проведенных с целью нахождения оптимальных режимов резания, обеспечивающих требуемое качество обработки поверхности.

\section{ВНЕДРЕНИЕ РОБОТИЗИРОВАННЫХ СИСТЕМ В СОВРЕМЕННОЙ ПРОМЫШЛЕННОСТИ}

Из-за высоких требований к эффективности и гибкости в современном промышленном производстве необходимость в автоматизированных и высокоточных операциях механической обработки увеличилась. В этом контексте использование промышленных роботов является привлекательным решением, так как приносит экономическую выгоду, обладает широкими возможностями для переналадки, имеет большую универсальность и гибкость в сравнении с обрабатывающими центрами с ЧПУ [1].

Развитие современного машино- и приборостроения, микро- и оптоэлектроники постоянно повышает требования к технологическим процессам прецизионной обработки деталей, требует улучшения качества обработки их поверхностей и увеличения производительности процессов. Это обусловлено тем, что в настоящее время изделия микро- и оптоэлектроники миниатюризируются, а также усложняется конфигурация деталей машиностроения. В связи с этим значительно возрастает роль финишных операций, основными из которых являются шлифование и полирование, обеспечивающих высокое качество поверхности деталей $[2,3]$.

В последние десятилетия промышленные роботы используются для выполнения широкого круга промышленных задач, таких как обработка материалов, сварка, сборка, транспортировка, окраска распылением и вспомогательные операции обра- ботки, такие как удаление заусенцев, полировка, шлифование. Однако только 3-4\% от общего числа промышленных роботов используются непосредственно для механической обработки. Основная причина заключается в возникновении вибраций при обработке из-за невысокой жесткости роботов. Появление новых роботов повышенной жесткости позволило преодолеть эту проблему, благодаря чему роботизированные системы становятся эффективней для применения в большинстве операций механической обработки, а сотрудники механических цехов с энтузиазмом используют роботов для определенных многоосевых фрезерных применений $[1,4,5]$.

Роботы имеют ряд технологических преимуществ:

$\rightarrow$ стоимость покупки и эксплуатации ниже, чем у станков с ЧПУ;

$\rightarrow$ большие кинематические возможности при обработке;

$\rightarrow$ более высокие скорости перемещений;

$\rightarrow$ большие возможности переналадки, что делает эффективным применение роботов в многономенклатурном производстве.

Известен также ряд технологических недостатков роботов:

$\rightarrow$ недостаточная жесткость;

$\rightarrow$ недостаточная точность позиционирования;

$\rightarrow$ сложность программирования.

Основная сложность в программировании промышленных роботов заключается в том, что 
каждый производитель роботов использует собственный проприетарный язык для написания управляющих программ, поскольку не существует единого стандарта. Этот факт послужил сильной мотивацией для ведущих мировых производителей роботов и программного обеспечения к разработке универсальных программных решений, например на основе G-кодов, чтобы приблизить процесс программирования робота к аналогичному процессу на станках ЧПУ, который уже многие годы применяется в промышленности.

В последние годы производства становятся все более многономенклатурными, сложность изделий увеличивается, а их партии уменьшаются. В таких условиях станки с ЧПУ имеют низкий коэффициент технического использования (около 50\%), длительность переналадок вызывает дополнительные простои и увеличивает срок окупаемости оборудования. Роботы полностью отвечают таким вызовам, лучше адаптируются к изменению конструкции деталей и новым операциям. Ограниченная рабочая зона и кинематика станков с ЧПУ вынуждают обрабатывать одну деталь за несколько операций и проводить несколько переустановок, что увеличивает производственный цикл. Роботизированные системы за счет своих технологических возможностей могут вести обработку за один установ и снять эти ограничения. В этой связи производители все чаще начинают задумываться о применении роботов вместо станков с ЧПУ $[3,4,6]$.

\section{РАЗНОВИДНОСТИ КОМПОНОВКИ РОБОТИЗИРОВАННЫХ СИСТЕМ}

Одна из основных проблем промышленных роботов - точность позиционирования - обусловлена двумя причинами: систематическими и случайными ошибками.

Систематические ошибки. Данную группу делят на систематические постоянные и систематические закономерно изменяющиеся погрешности. Систематические постоянные погрешности устраняются калибровкой робота. Систематические закономерно изменяющиеся погрешности связаны с износом инструмента, также они могут быть связаны с проявлением теплового фактора.

Случайные погрешности. Основные причины возникновения данных погрешностей обусловлены низкой жесткостью робота-манипулятора, факторами окружающей среды, ограниченной пропускной способностью контроллера и трением в суставах робота. Силы резания, действующие на выходное звено, могут смещать руку манипулятора, приводя к ошибке положения. Жесткость промышленных роботов-манипуляторов в 50-100 раз меньше, чем станков с ЧПУ, и из-за сил резания на выходном звене могут возникать вибрации [7].

Необходимость учета факторов, влияющих на точность положения выходного звена робота, создает необходимость это положение отслеживать в ходе выполнения операции обработки поверхности. С этой целью в промышленных роботах используют устройство контроля силы взаимодействия с деталью для возможности онлайн-корректировки траектории движения на основе полученных данных о силах резания. Необходимость подобной системы при выполнении механической обработки обусловлена также следующими причинами:

$\rightarrow$ износом инструмента, вызывающим отклонение от требуемых геометрических размеров;

$\rightarrow$ случайным положением и размерами погрешностей на поверхности заготовки;

$\rightarrow$ сложностью формы детали, затрудняющей программирование робота.

Устройства силового контроля позволяют обеспечить постоянство усилий при обработке, что позволяет адаптировать систему к отклонениям между запрограммированной и фактической траекториями на поверхности заготовки и повысить качество обработки. Процесс полировки поверхности требует высокого качества, чего невозможно достичь без постоянства усилий между инструментом и деталью, и постоянной скорости подачи. Износ инструмента может быть сведен к минимуму, а поломки инструмента можно избежать, если силы резания постоянны. Учитывая потребность современной промышленности, все больше производителей роботов предлагают различные возможности использования позиционно-силового контроля $[8,9]$.

Следующим шагом при создании операции механической обработки с использованием промышленного робота является выбор компоновки роботизированной ячейки. Можно выделить два основных технических решения.

1. Деталь закреплена на роботе (рис. 1) [10], а привод инструмента закреплен на неподвижном основании. Данный способ используется, когда обрабатываемые детали имеют небольшие размеры и вес, а инструмент требует мощного привода.

2. Инструмент осевой небольшого диаметра (сверло, фреза) вместе со шпинделем установлен на выходном звене робота (рис. 2) [1], а деталь имеет значительные габариты (например, корпусные детали, сварные конструкции). Данное решение обеспечивает большую гибкость за счет возможности доступа к большему числу поверхностей детали, в том числе внутренних или расположенных под углом. 


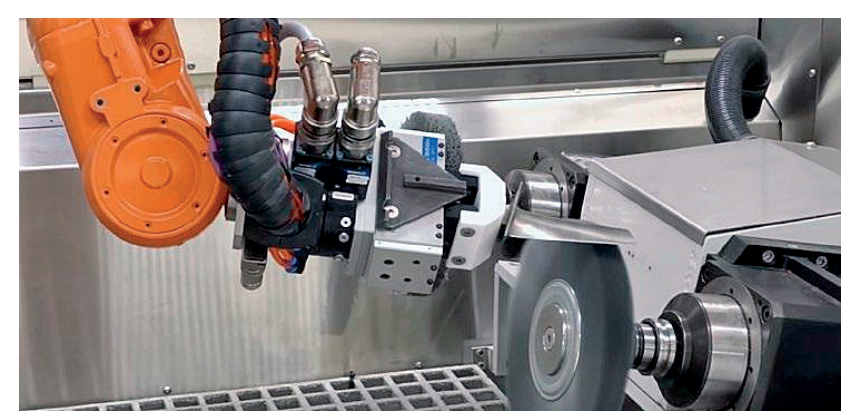

Puc. 1. Обработка с закреплением заготовки на роботе

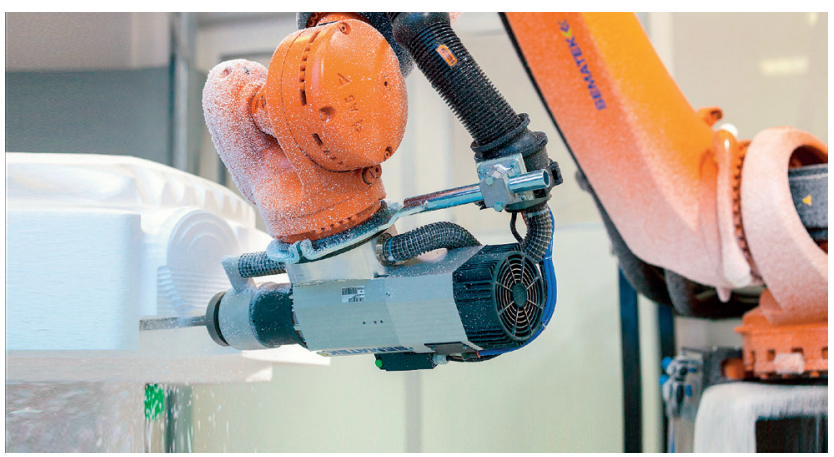

Puc. 2. Обработка с закреплением инструмента на роботе

Выбрав компоновку роботизированной ячейки, необходимо окончательно определить ее основные элементы. В состав роботизированной ячейки входят:

1. Промышленный робот. Из большого многообразия устройств необходимо выбрать робот, габариты и рабочая область которого позволяют производить обработку всей номенклатуры деталей, подлежащих обработке на производстве. Основными характеристиками промышленного робота для механической обработки являются:

$\rightarrow$ максимально достижимая точность позиционирования;

$\rightarrow$ длительность цикла, зависящая от конструкции и размеров робота;

$\rightarrow$ рабочий диапазон робота и устройства для его монтажа, оптимальное сочетание которых позволит обеспечить доступ к зоне обработки;

$\rightarrow$ защита робота от неблагоприятных условий, позволяющая обезопасить промышленный робот от мелкой пыли и стружки.

2. Шкаф управления. Система управления отвечает за управление перемещениями робота, обеспечение обратной связи, передачу данных о параметрах резания и корректировку траектории, а следовательно, и за качество обработки.

3. Силомоментный датчик. Шестиосевые датчики сил и моментов дают роботам тактильное ощущение прикосновения, посылая обратную связь по силе и моменту для контроля положения робота. Датчик силы и момента измеряет шесть составляющих $\left(F_{x}, F_{y}, F_{z}, T_{x}, T_{y}\right.$ и $\left.T_{z}\right)$. Сигналы обрабатываются непосредственно датчиком и передаются с помощью различных протоколов обмена данными.

4. Шпиндель. В зависимости от целевого использования, выполняемых операций и обрабатываемых материалов можно выбрать шпиндель с различными характеристиками (мощность, максимальное число оборотов, частота, тип охлаждения и вес). Вес шпинделя имеет большое значение, так как монтируется на выходном звене робота. Грузоподъемность робота ограничена, а большая нагрузка приведет к снижению точности позиционирования робота. Дополнительную нагрузку дает технологическая оснастка и инструмент.

\section{5. Инструмент и ограждения рабочей зоны} робота.

В качестве дополнительной опции возможно применение промышленного пылесоса или системы высокого вакуума для удаления мелкодисперсной пыли из зоны обработки. Вакуумная установка с прямым приводом может быть оборудована функциями автоматического запуска/остановки и очистки фильтра, также имеет рукавные фильтры для тонкодисперсной пыли.

\section{ЭКСПЕРИМЕНТАЛЬНЫЕ ИССЛЕДОВАНИЯ ОПТИМИЗАЦИИ РЕЖИМОВ РЕЗАНИЯ ПРИ РОБОТИЗИРОВАННОЙ ОТДЕЛОЧНОЙ ОБРАБОТКЕ}

Получение высокого качества поверхности после обработки и соответствие геометрических размеров требованиям чертежа являются обязательными при механической обработке, а их достижение возможно при высокой точности позиционирования робота и отсутствии отклонений от требуемой траектории движения инструмента. Это достижимо, если силы резания не превышают допустимых значений, приводящих к отклонению траектории робота. Для минимизации сил резания и вибраций при обработке необходимо проводить исследования, которые позволят установить оптимальные технологические режимы для конкретной операции.

В данной работе исследование проводилось на барабане колеса вертолета (рис. 3). Деталь изготавливают из магниевого сплава МЛ5ПЧ за одно целое с ободом, ступицей и одной ребордой. На барабане монтируется камера и покрышка, которая является силовым элементом. Она воспринимает нагрузки и передает их на барабан колеса.

Использование промышленного робота планируется в технологическом процессе ремонта барабана, вместо ручной операции зачистки поверхно- 
сти. Согласно заводской ремонтной технологии, барабан проходит операцию дробеструйной обработки, для чего используется дробь из нержавеющей стали диаметром 0,2 мм. Такая обработка позволяет получить показатель Ra, равный 6,3 мкм. Данная величина шероховатости является окончательной для большей части поверхностей колеса и не требует дальнейшей обработки. Однако шероховатость наружных поверхностей, по которым происходит прилегание камеры колеса, должна быть улучшена до величины параметра шероховатости Ra, равного 1,6 мкм. С этой целью использование промышленных роботов, обладающих высокой гибкостью в производстве, является эффективным вариантом, так как номенклатура завода состоит из большого числа колес различных типоразмеров, что обеспечивает большую программу выпуска.

Учитывая габариты и конструкцию барабана, целесообразно его разместить на столе, а обработку вести инструментом, закрепленным на роботе. Это обеспечит более высокую точность позиционирования, за счет меньшей нагрузки на выходное звено робота, и более высокое качество обработки.

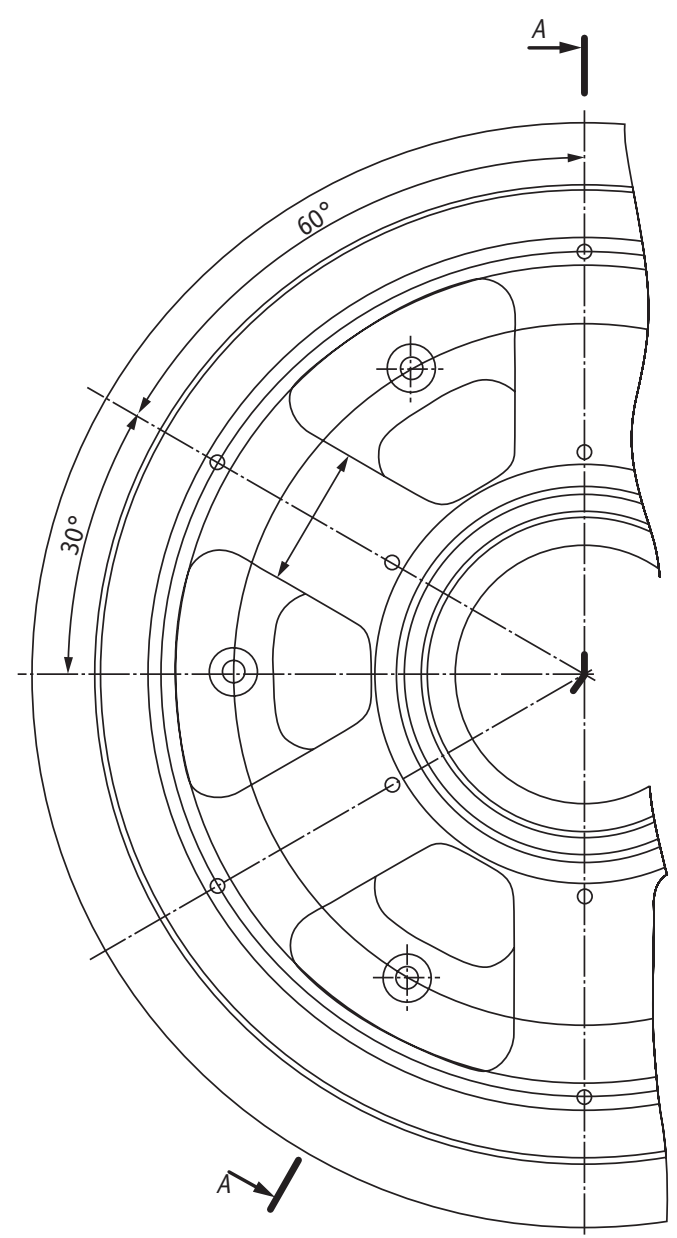

Учитывая размер обрабатываемых поверхностей и тип крепления в шпинделе, в качестве инструмента для обработки выбран шлифовальный круг, который крепится на опорный диск с помощью Velcroподложки. Такая система обеспечивает быструю смену кругов различной зернистости и быстрое продолжение работы.

Для проведения исследований в лаборатории «Промышленные роботы» Московского политехнического университета была создана экспериментальная установка на основе промышленного робота IRB-140 компании ABB (рис. 4). Это универсальный робот, точность позиционирования которого составляет $\pm 0,03$ мм; грузоподъемность - до 6 кг; радиус активного действия - 810 мм. Для осуществления обработки робот был дооснащен шпинделем модели GDF65-24Z/0.8. Шпиндель имеет следующие технические характеристики:

$\rightarrow$ мощность $-0,8$ кВт;

$\rightarrow$ крутящий момент $-0,22 \mathrm{H} \cdot \mathrm{M}$;

$\rightarrow$ максимальная частота вращения - 24000 об/мин;

$\rightarrow$ частота тока - 400 Гц;

$\rightarrow$ вес -3 кг.

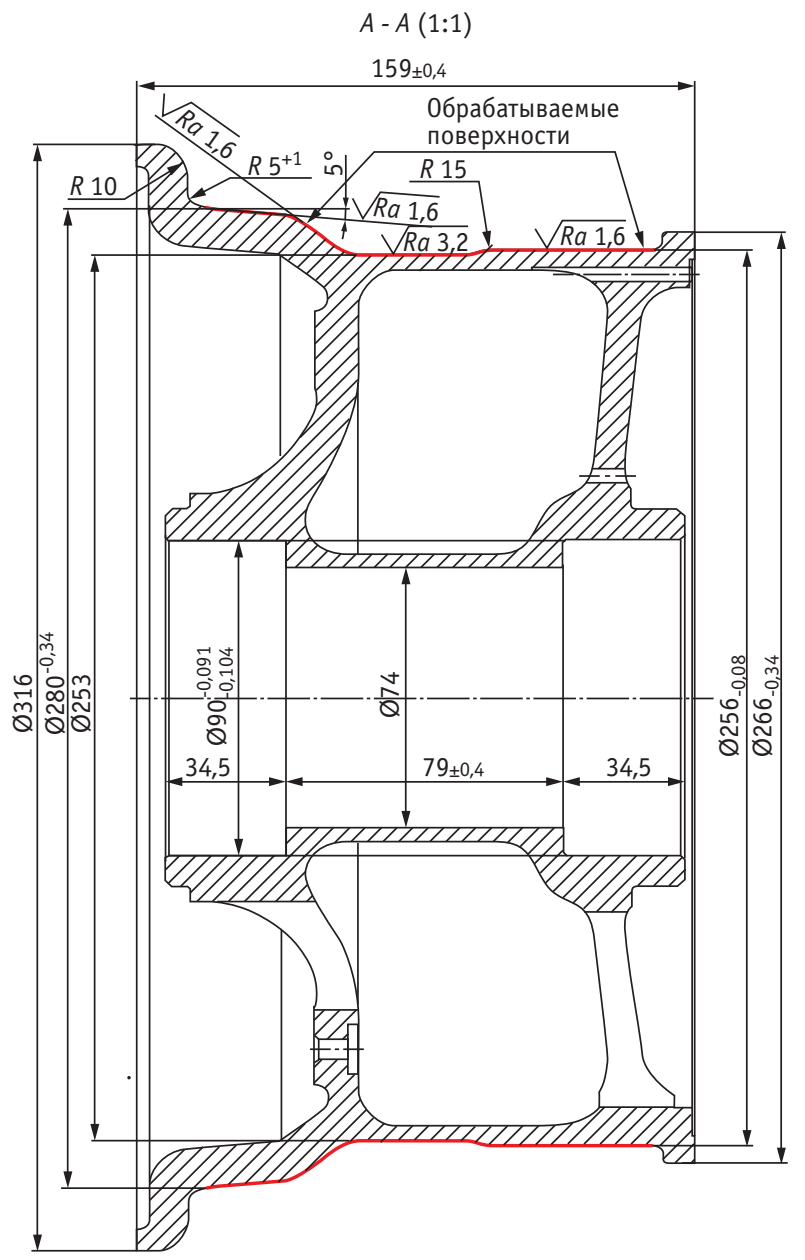

Puc. 3. Барабан колеса вертолета 
Таблица 1. Факторы, влияющие на процесс обработки

\begin{tabular}{|l|c|}
\hline Фактор & 0бозначение \\
\hline Частота оборотов шпинделя, $n$, об/мин & $x_{1}$ \\
\hline Подача, S, м/мин & $x_{2}$ \\
\hline Глубина резания, $t$, мм & $x_{3}$ \\
\hline Зернистость инструмента, $z$ & $x_{4}$ \\
\hline
\end{tabular}

Таблица 2. Уровни факторов и интервалы варьирования

\begin{tabular}{|l|c|c|c|c|c|}
\begin{tabular}{l|c|c|c|} 
Факто- \\
ры
\end{tabular} & \multicolumn{3}{|c|}{ Уровни } & $\begin{array}{c}\text { Интервал } \\
\text { варьирования }\end{array}$ & $\begin{array}{c}\text { Размер- } \\
\text { ность }\end{array}$ \\
\hline$x_{1}$ & 9600 & 10800 & 12000 & 1200 & об/мин \\
\hline$x_{2}$ & 0,5 & 1,0 & 1,5 & 0,5 & м/мин \\
\hline$x_{3}$ & 0,3 & 0,2 & 0,1 & 0,1 & мм \\
\hline$x_{4}$ & 120 & 240 & 320 & 120 & - \\
\hline
\end{tabular}

Для крепления шпинделя на роботе была спроектирована и изготовлена специальная оснастка. Кроме шпинделя на оснастке устанавливался силомоментный датчик Schunk FT AXIA. Данный датчик уже нашел применение в ряде технологических задач, в том числе при шлифовании и сборке. Датчик делает возможным онлайн-получение данных о силе резания и влиянии различных факторов на процесс.

Для анализа данных в исследовании был выбран полнофакторный эксперимент (ПФЭ) [11, 12]. При его проведении учитывались наиболее значимые факторы, возникающие в процессе резания (табл. 1).
Были определены выходные параметры, характеризующие протекание процесса роботизированной отделочной обработки: $\mathrm{y}_{1}\left(P_{x}\right)$ - составляющая силы резания по оси $X, H ; \mathrm{y}_{2}\left(P_{y}\right)$ - составляющая силы резания по оси $\mathrm{У}, \mathrm{H} ; \mathrm{y}_{3}\left(P_{z}\right)$ - составляющая силы резания по оси $Z, H ; y_{4}\left(T_{x}\right)$ - момент, действующий по оси $X, H \cdot$ м.

Уровни факторов и интервалы варьирования представлены в табл. 2.

Число экспериментов зависит от количества факторов и в данном эксперименте равнялось 16. С каждым набором факторов опыт был проведен пять раз для определения средних значений, что позволило исключить случайное влияние внешних воздействий.

Математическая модель эксперимента имела вид полинома первой степени:

$$
y=b_{0}+\sum_{i=1}^{n} b_{i} \cdot x_{i}+\sum_{i \neq 1} b_{i l} \cdot x_{i} \cdot x_{l} \ldots
$$

После проведения анализа значимости коэффициентов выявлено, что значимыми являются следующие коэффициенты:

$\rightarrow$ для $y_{1}: b_{0}, b_{3}, b_{13}, b_{23}$;

$\rightarrow$ для $y_{2}: b_{0}, b_{1}, b_{3}, b_{4}, b_{12}$;

$\rightarrow$ для $y_{3}: b_{0}, b_{1}, b_{23}, b_{24}$;

$\rightarrow$ для $y_{4}: b_{0}, b_{2}, b_{23}$.

Приведя полученные выражения к линейному виду, получили:

$\rightarrow P_{x}=54,7+3,56 \cdot t-0,31 \cdot n \cdot t-0,43 \cdot S \cdot t$,

$\rightarrow P_{y}=38,6+9,5 \cdot n-10,75 \cdot t-6 \cdot z+2,37 \cdot n \cdot S$,

$\rightarrow P_{z}=31,6+7,31 \cdot n+8,06 \cdot S \cdot t+0,31 \cdot S \cdot z$,

$\rightarrow T_{x}=2,6+0,06 \cdot S+0,18 \cdot S \cdot t$.

Проведенный полнофакторный эксперимент (ПФЭ) показал, что с вероятностью 0,95 математи-
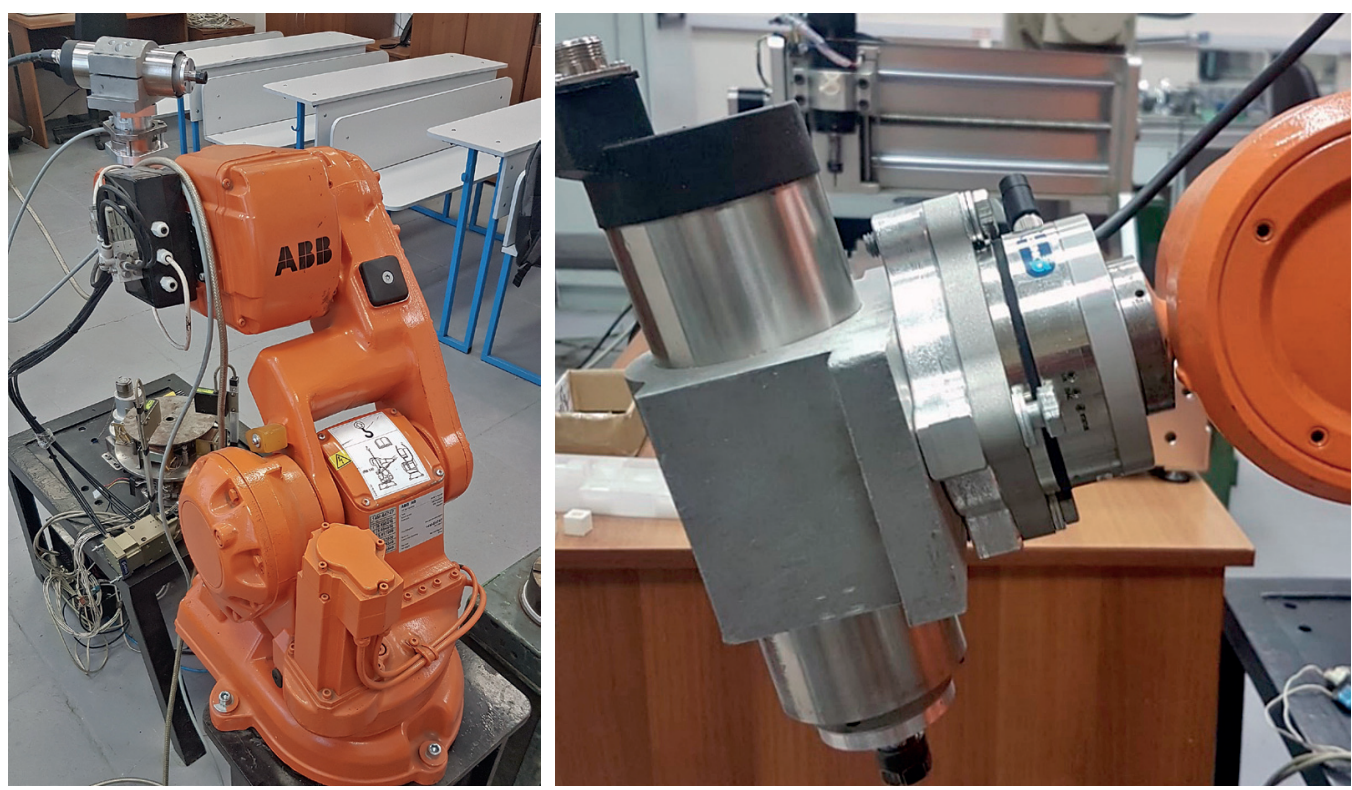

Puc. 4.

Экспериментальная установка в лаборатории «Промышленные роботы» 
ческая модель может считаться адекватной экспериментальным данным, а значит, на основе этих данных можно принимать дальнейшие решения и использовать ПФЭ при анализе процесса роботизированной отделочной обработки.

После проведения полнофакторного эксперимента и выбора оптимальных режимов резания для данного материала детали, которые обеспечили наименьшие силы резания и вибрации, были произведены замеры величин параметра шероховатости Ra. Для определения величины использовался портативный профилометр модели MarSurf PS1 со встроенным калибром шероховатости. Измерения показали, что стабильность достижения требуемой величины параметра шероховатости обеспечивается при использовании кругов с показателем зернистости Р320 по ГОСТ 52381-2005.

\section{ЗАКЛЮЧЕНИЕ}

Ручные слесарные операции широко распространены в машиностроении, но повышение требований к условиям труда, качеству и гибкости производства приводит к активному внедрению современных технологий, таких как роботизированные системы.

Проведенные исследования показали техническую возможность применения роботов для отделочных операций обработки колеса вертолета.

Выполненные исследования доказали применимость позиционно-силового управления роботом на основе применения силомоментного датчика. Однако для каждой технологической операции должны быть разработаны специализированные алгоритмы управления по критерию удельного съема материала или аналогичному критерию. Создание подобных алгоритмов является основной научной составляющей данной работы.

Регулирование сил резания дает значительные преимущества, повышая производительность и улучшая качество поверхности деталей. Перспективным в настоящее время считается управление удельным съемом материала по оценке сил резания и варьированию подачи. При этом возможно применение различных стратегий управления, включая пропорционально-интегрально-дифференцирующий регулятор (PID), адаптивное управление и использование нечеткой логики в зависимости от технологических особенностей операции.

\section{ЛИТЕРАТУРА}

1. Milutinovic D., Glavonjic M., Slavkovic N. Reconfigurable tobotic machining system controlled and programmed in a machine tool manner // Int J Adv Manuf Technology. 2011. PP. 1217-1229.

2. Пилиневич Л.П. Пористый алмазный инструмент с анизотропной поровой структурой для шлифо- вально-полировальных работ // Доклад БГУИР. 2016. № 3 (97). C. 44-48.

3. Pandremenos J., Doukas C., Stavropoulos P., Chryssolouris G. Machining with robots: a critical review - 7th International Conference on Digital Enterprise Technology, Athens, Greece, 2011.

4. Вартанов М.В., Зинина И.Н., Зотин Д.О. Технологические возможности роботизированной отделочной обработки деталей в условиях многономенклатурного производства // Вестник РГАТУ. 2017. № 1 (40). С. 190-193.

5. Zaghbani I., Lamraou M., Songmene V., Thomas M. el Badaoui M. Robotic High Speed Machining of Aluminum Alloys - Proceedings of the 4th edition of the International Conference on High Speed Machining (ICHSM'2010), Harbin, China, 2011.

6. Sörnmo O., Olofsson B., Schneider U., Robertsson A., Johansson R. Increasing the Milling Accuracy for Industrial Robots Using a Piezo-Actuated HighDynamic Micro Manipulator. - IEEE/ASME International Conference on Advanced Intelligent Mechatronics (AIM), 2012.

7. Seong Hyeon K., Eunseok N., Soon-Hong $\mathbf{H}$. Robotic Machining: A Review of Recent Progress // International Journal of Precision Engineering and Manufacturing. 2019. PP. 1629-1642.

8. Krantz M., Andersson R. Robotized Polishing and Deburring with Force Feedback Control // Master Thesis, Master Degree program in Robotics University West, Department of Engineering Science, S-461 86 Trollhättan, SWEDEN, 2010.

9. Мисюров М.Н. Силовое управление роботами и его применение для удаления заусенцев промышленных деталей // Молодой ученый. 2016. № 3 (107). С. 155-158.

10. Live demonstration at EMO 2015, Milan, Italy in Hall 4, stand C05, from Delcam Professional Services

11. Белова Н.В., Малинина О.А. Применение полного фракторного эксперимента при измерении параметров газового потока // Издательство молодой ученый. № 4 (15). 2010. С. 65-70.

12. Кристаль М.Г., Горелова А.Ю. Обработка результатов планирования экстремального эксперимента: учебное пособие. - Волгоград: ВолГТУ, 2019. 70 с.

\section{ВАРТАНОВ Михаил Владимирович -}

доктор технических наук, профессор кафедры «Технологии и оборудование машиностроения» Московского политехнического университета

\section{ВЛАСОВ Алексей Сергеевич -}

магистрант кафедры «Технологии и оборудование машиностроения» Московского политехнического университета 